\title{
Front Matter: Volume 10586
}

, "Front Matter: Volume 10586," Proc. SPIE 10586, Advances in Patterning Materials and Processes XXXV, 1058601 (11 May 2018); doi:

$10.1117 / 12.2323987$

SPIE. Event: SPIE Advanced Lithography, 2018, San Jose, California, United States 


\section{PROCEEDINGS OF SPIE}

\section{Advances in Patterning Materials and Processes XXXV}

Christoph K. Hohle

Editor

26 February-1 March 2018

San Jose, California, United States

Sponsored by

SPIE

Cosponsored by

Applied Materials, Inc. (United States)

Published by

SPIE 
The papers in this volume were part of the technical conference cited on the cover and title page. Papers were selected and subject to review by the editors and conference program committee. Some conference presentations may not be available for publication. Additional papers and presentation recordings may be available online in the SPIE Digital Library at SPIEDigitalLibrary.org.

The papers reflect the work and thoughts of the authors and are published herein as submitted. The publisher is not responsible for the validity of the information or for any outcomes resulting from reliance thereon.

Please use the following format to cite material from these proceedings:

Author(s), "Title of Paper," in Advances in Patterning Materials and Processes XXXV, edited by Christoph K. Hohle, Proceedings of SPIE Vol. 10586 (SPIE, Bellingham, WA, 2018) Seven-digit Article CID Number.

ISSN: 0277-786X

ISSN: 1996-756X (electronic)

ISBN: 9781510616646

ISBN: 9781510616653 (electronic)

Published by

SPIE

P.O. Box 10, Bellingham, Washington $98227-0010$ USA

Telephone +1 3606763290 (Pacific Time) · Fax +1 3606471445

SPIE.org

Copyright (C) 2018, Society of Photo-Optical Instrumentation Engineers.

Copying of material in this book for internal or personal use, or for the internal or personal use of specific clients, beyond the fair use provisions granted by the U.S. Copyright Law is authorized by SPIE subject to payment of copying fees. The Transactional Reporting Service base fee for this volume is $\$ 18.00$ per article (or portion thereof), which should be paid directly to the Copyright Clearance Center (CCC), 222 Rosewood Drive, Danvers, MA 01923 . Payment may also be made electronically through CCC Online at copyright.com. Other copying for republication, resale, advertising or promotion, or any form of systematic or multiple reproduction of any material in this book is prohibited except with permission in writing from the publisher. The CCC fee code is 0277 $786 \mathrm{X} / 18 / \$ 18.00$.

Printed in the United States of America.

Publication of record for individual papers is online in the SPIE Digital Library.

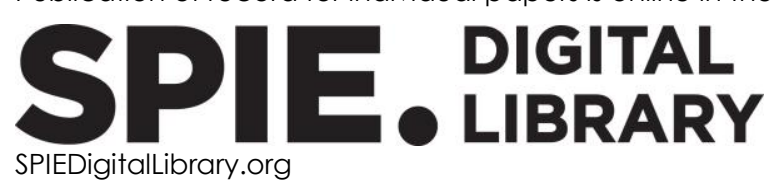

Paper Numbering: Proceedings of SPIE follow an e-First publication model. A unique citation identifier (CID) number is assigned to each article at the time of publication. Utilization of CIDs allows articles to be fully citable as soon as they are published online, and connects the same identifier to all online and print versions of the publication. SPIE uses a seven-digit CID article numbering system structured as follows:

- The first five digits correspond to the SPIE volume number.

- The last two digits indicate publication order within the volume using a Base 36 numbering system employing both numerals and letters. These two-number sets start with $00,01,02,03$, 04, 05, 06, 07, 08, 09, OA, OB ... 0Z, followed by 10-1Z, 20-2Z, etc. The CID Number appears on each page of the manuscript. 


\title{
Contents
}

\author{
vii Authors \\ xi Conference Committee
}

EUV: RESIST PROCESSES: JOINT SESSION WITH CONFERENCES 10583 AND 10586

1058604 Aqueous developers for positive tone ultrathin chemically amplified EUV resists [10586-3]

1058605 EUV via hole pattern fidelity enhancement through novel resist and post-litho plasma treatment [10586-4]

1058606 EUV resist sensitization and roughness improvement by PSCAR ${ }^{\text {TM }}$ with in-line UV flood exposure system [10586-5]

EUV: METAL-BASED RESISTS: JOINT SESSION WITH CONFERENCES 10583 AND 10586

1058607 Surface characterization of tin-based inorganic EUV resists [10586-6]

1058608 Mechanisms of photodecomposition of metal-containing EUV photoresists: isotopic labelling studies [10586-7]

MATERIALS AND ETCH INTEGRATION: JOINT SESSION WITH CONFERENCES 10586 AND 10589

10586 OA Ultimate edge-placement control using combined etch and lithography system optimizations [10586-9]

EUV: FUNDAMENTALS

10586 OC Unraveling the role of photons and electrons upon their chemical interaction with photoresist during EUV exposure (Invited Paper) [10586-1 1]

10586 OD Polymer effects on PAG acid yield in EUV resists [10586-12]

10586 OE Characterization of metal resist for EUV lithography using infrared free electron laser [10586-13]

10586 OF Investigations on EUVL metal resist dissolution behavior using in situ high speed atomic force microscopy [10586-14] 
EUV: NOVEL PROCESSES

10586 OG Novel EUV resist materials for $7 \mathrm{~nm}$ node and beyond [10586-15]

$10586 \mathrm{OH}$ Study of electron beam and extreme ultraviolet resist utilizing polarity change and radical crosslinking [10586-16]

$1058601 \quad$ Polymer brush as adhesion promoter for EUV patterning [10586-17]

10586 0J LWR enhancement for 300mm track processing [10586-18]

10586 OK Novel Sn-based photoresist for high aspect ratio patterning [10586-19]

\section{HARDMASKS AND UNDERLAYER}

$10586 \mathrm{OL}$ The development of an SC1 removable SiARC [10586-20]

10586 OM New spin on carbon materials made from hemicellulose for hardmask layer [10586-21]

10586 ON High-carbon fullerene based spin-on organic hardmask [10586-22]

DSA MATERIALS AND CHARACTERIZATION: JOINT SESSION WITH CONFERENCES 10586 AND 10584

10586 OP Utilization of metal-polymer interactions for self-aligned directed self-assembly of device relevant features [10586-24]

10586 OQ Pillars fabrication by DSA lithography: material and process options [10586-25]

DSA: DEFECTIVITY AND HIGH-CHI

10586 OU A simulation study on bridge defects in lamellae-forming diblock copolymers [10586-30]

10586 OV Directed self-assembly of triblock copolymers for sub-10nm nanofabrication using polymeric additives [10586-31]

10586 OW Straightforward directed self-assembly process flows enabled by advanced materials [10586-32]

DSA: CYLINDER FORMING BCP AND METROLOGY

$105860 Z$ Automated lamellar block copolymer process optimization with machine learning [10586-35]

iv 
1058611 Multi-color fly-cut-SAQP for reduced process variation [10586-37]

1058612 Thick photosensitive polyimide film side wall angle variability and scum improvement for IC packaging stress control [10586-38]

1058613 Resist-polymer ablation by mid-infrared-free-electron laser [10586-39]

1058615 Evaluation of anti-sticking layers performances for $200 \mathrm{~mm}$ wafer scale Smart NIL ${ }^{\mathrm{TM}}$ process through surface and defectivity characterizations [10586-41]

POSTER SESSION

1058616 Application specific ratings of filters for negative-tone developer [10586-42]

1058617 The intrinsic role of membrane morphology to reduce defectivity in advanced photochemicals [10586-43]

1058618 Targeted removal of metallic contamination from lithography solvents using membrane purifiers [10586-44]

10586 1A Improved airborne molecular contaminant filter performance for photolithography [10586-46]

10586 1D Directed Self-Assembly (DSA) for contact applications [10586-49]

10586 1E Fast annealing DSA materials designed for sub-5 nm resolution [10586-59]

10586 IF Desirable material selection on self-aligned multi-patterning [10586-50]

$105861 G$ Synthesis of metal nanoparticle and patterning in polymeric films induced by electron beam [10586-51]

$10586 \mathrm{1H} \quad$ Mechanism study of ion implantation on photoresist shrinkage [10586-52]

$1058611 \quad$ Chemical trimming overcoat: an advanced composition and process for photoresist enhancement in lithography [10586-58]

10586 1K Development of new polyphenols applied to spin-on carbon hardmask with characteristics of high-heat resistance and good planarization [10586-54]

10586 IM Effects of fluorine contamination on spin-on dielectric thickness in semiconductor manufacturing [10586-56]

$105861 \mathrm{~N}$ Investigating the threshold electron energy for reactions in EUV resist materials [10586-53]

1058610 Nanoscale inhomogeneity and photoacid generation dynamics in extreme ultraviolet resist materials [10586-60] 
10586 IP Molecular organometallic resists for EUV (MORE): reactivity as a function of metal center (Bi, Sb, and Te) [10586-61]

Proc. of SPIE Vol. $105861058601-6$

Downloaded From: https://www.spiedigitallibrary.org/conference-proceedings-of-spie on 26 Apr 2023 Terms of Use: https://www.spiedigitallibrary.org/terms-of-use 


\section{Authors}

Numbers in the index correspond to the last two digits of the seven-digit citation identifier (CID) article numbering system used in Proceedings of SPIE. The first five digits reflect the volume number. Base 36 numbering is employed for the last two digits and indicates the order of articles within the volume. Numbers start with 00, 01, 02, 03, 04, 05, 06, 07, 08, 09, OA, OB...0Z, followed by 10-1Z, 20-2Z, etc.

\author{
Aizawa, Ryo, 06 \\ Amat, E., $O Q$ \\ Araki, Mitsunori, 13 \\ Arceo de la Pena, Abraham, ol \\ Arisawa, You, $\mathrm{OM}$ \\ Asai, Masaya, OJ, OM \\ Ayothi, Ramakrishnan, 0 I \\ Azuma, Tsukasa, OU \\ Baranowski, Paul, 1 I \\ Belloni, Jacqueline, $1 G$ \\ Bernard, G., OZ \\ Biesemans, Serge, 06 \\ Biolsi, Peter, 11 \\ Bos, S., 15 \\ Brainard, Robert L., 08, 0D, 1N, IP \\ Brown, Alan G., ON \\ Calderas, Eric, OW \\ Cameron, Jim, OL \\ Carcasi, Michael, 06 \\ Cattani, Giordano, OA \\ Cayrefourcq, Ian, OQ, 15 \\ Chandonait, Jonathan, OD, IN \\ Chang, Ching-Yu, 1D, 10 \\ Chang, Vencent, 10 \\ Chen, Wei-Chi, 10 \\ Chen, Xuanxuan, OV \\ Cheng, Joy, 10 \\ Cheng, Yuan-Chung, 10 \\ Chevalier, Xavier, 0Z, 15 \\ Cho, Jae Kyu, 12 \\ Choi, Peter, 05 \\ Clark, Michael B., Jr., OL \\ Coley, Suzanne, OL \\ Corliss, Daniel, 0 I \\ Cui, Li, OL \\ Cutler, Charlotte, OL \\ Daugherty, Richard, OW \\ Dawson, Guy, ON \\ Dazai, Takahiro, $1 F$ \\ Dei, Satoshi, 06 \\ Delachat, F., 15 \\ Denbeaux, Greg H., 08, 0D, 1N, 1P \\ Deng, Hai, 1E \\ Dervilllé, A., $0 Z$ \\ De Silva, Anuja, 0 \\ De Simone, Danilo, 06, 0C \\ DeVilliers, Anton, 11 \\ Diulus, J. Trey, 07 \\ Dixit, Girish, OA
}

Dolejsi, Moshe, OP

Drent, Waut, 0J

Duggan, Mark, 12

Echigo, Masatoshi, $1 \mathrm{~K}$

Enomoto, Masashi, 06

Enomoto, Satoshi, $\mathrm{OH}$

Fang, Zhou, $1 \mathrm{H}$

Farrell, Richard A., 11

Faruqui, Danish, 12

Feldman, Leonard C., OK

Felix, Nelson M., Ol

Feng, Mu, 05

Fernandez-Regulez, M., $0 Q$

Fitzgibbons, Thomas C., OL

Fonseca, Carlos, 05

Foubert, Philippe, 06

Foucher, J., 0 Z

Fournel, F., 15

Franke, Elliott, 11

Frederick, Ryan T., 07

Fujimaki, Nishiki, OG

Fujimori, Toru, OG

Fujita, Mitsuhiro, OG

Furukawa, Tsuyoshi, 이

Furutani, Hajime, OG

Garfunkel, Eric L., OK

Gharbi, A., OQ

Gibbons, Sean, OD, 1N

Goldfarb, Dario L., 04

Greene, Daniel, OL

Grzeskowiak, Steven, 08, 0D, 1N, 1P

Guerrero, Douglas, OW

Guo, Jing, Ol

Gustafsson, Torgny, OK

Halder, Sandip, OA

Hamzik, James, 18

Harumoto, Masahiko, 0J, OM

Hayakawa, Makoto, 06

Hellin, David, OA

Herman, Gregory S., 07

Hetzer, David, 11

Hoang, Brian, $1 \mathrm{~A}$

Hockey, Mary Ann, OW

Hong, Soonsang, $1 \mathrm{M}$

Hongo, Koki, OM

Hori, Masafumi, 06

Horiuchi, Junya, $1 \mathrm{~K}$

Hotalen, Jodi, 11

Hou, Xisen, 11 
Hsieh, Ken-Hsien, 1D

Huang, Yuan-Chien, 1D

Hutchison, Danielle C., 07, OK

Hwang, Sung Min, 1M

Ide, Hiroyuki, 06

Iguchi, Naoya, 16

Imai, Takayuki, OE, 13

Itani, Toshiro, OE, OF

Ito, Kiyohito, 05

Jaber, Jad, 18

Jaenen, Patrick, OA

Jee, Tae Kwon, 05

Kamei, Yuya, 06

Kaminsky, Jake, OD, 1N

Kandel, Yudhishthir, IN

Kasahara, Yusuke, OU

Kaur, Irvinder, 11

Kaushik, Kumar, 05

Kawada, Yukihisa, 16

Kawasaki, Takayasu, OE, 13

$\mathrm{Ke}$, lou-Sheng, OL

Kim, Hyoung-ryeun, 1M

Kim, Samyoung, 1M

Kimura, Toru, 06

Ko, Akitero, 11

Ko, Tsung-Han, 1D

Kodera, Katsuyoshi, OU

Kohyama, Tetsu, 17, 18

Koike, Kyohei, 05, $1 \mathrm{~F}$

Kondo, Yoshihiro, 06

Konishi, Yoshitaka, 06

Kozawa, Takahiro, $\mathrm{OH}, 1 \mathrm{G}$

Kubis, Michael, OA

Kumar, Bharat, 04

LaBeaume, Paul, OL

Lada, Tom, ON

Lais, Joshua, $1 \mathrm{~A}$

Larrey, V., 15

Lee, Chih-Jie, 1D

Lee, Chung-Ju, ID

Leonard, JoAnne, OL

Leray, Philippe, OA

Leusink, Gert, 11

$\mathrm{Li}, \mathrm{CUi}, \mathrm{OL}$

Li, Jiajing, OV

Li, Mengjun, OK

Li, Mingqi, 1 I

Li, Xuemiao, $1 \mathrm{E}$

Liang, Yichen, OW

Lin, Chin-Hsiang, 1D

Lin, John, 10

Liv, Cong, 11

Longenbach, Travis, 12

Luca, Melisa, OA

Ly, Saksatha, 18

Lyubinetsky, Igor, 07

Machida, Kohei, $\mathrm{OH}$

Makinoshima, Takashi, IK

Manichev, Viacheslav, OK

Marignier, Jean-Louis, IG
Maruyama, Ken, 06

Maslow, Mark, 05

Masuda, Kazushi, 16

McClelland, Alexandra, ON

Mehta, Sohan Singh, 12

Meli, Luciana, 0 I

Meliorsz, Balint, 06

Melvin, Lawrence S., III, IN

Metzler, Dominik, Ol

Mignot, Yann, 01

Minekawa, Yukie, 06

Mirza, Fahad, 12

Miura, Kozue, 17

Miyagi, Ken, OU

Miyake, Masayuki, 06

Mokhlespour, Salman, OA

Montgomery, Warren, ON

Morgan, Justin, 12

Morikita, Shinya, 05

Morita, Kazuyo, OM

Moriya, Teruhiko, 06

Mosfavi, Mehran, $1 G$

Murakami, Tetsuya, 16

Murphy, Michael, 08, 0D, IP

Nafus, Kathleen, 06

Nagahara, Seiji, 06

Nagai, Tomoki, 06

Nakagawa, Hisashi, 06

Nakashima, Hideo, 06

Nakayama, Chisayo, 0J, OM

Naruoka, Takehiko, 06

Navarro, Christophe, $0 Q, 15$

Nealey, Paul, OP, OV

Nicolet, Célia, 0Q, 15

Nihashi, Wataru, OG

Nyman, May, 07, OK

Oh, Changyeol, $1 \mathrm{M}$

Ohmori, Katsumi, $1 \mathrm{~F}$

Ohyashiki, Yasushi, 17

Oka, Hironori, 0G

Olsen, Morgan R., 07

Omatsu, Tadashi, OG

O'Meara, David, 11

Ongayi, Owendi, OL

Oshima, Akihiro, 06

Pain, L., OQ

Park, Jong, 11

Peng, $Y U, 1 E$

Perego, M., OQ

Perez-Murano, F., OQ

Petersen, John S., 06, OC

Peterson, Brennan, OA

Phillipe, J.-C., 15

Pieczulewski, Charles, 0J

Pimenta-Barros, P., OQ

Pollentier, Ivan, OC

Popere, Bhooshan, $\mathrm{OL}$

Premachandran, C. S., 12

Rabie, Mohamed, 12

Raley, Angelique, 11 
Raman, Thiagarajan, 12

Rathore, Ashish, OC

Reidy, Sean, 12

Reijnen, Liesbeth, 05

Reynaud, G., OQ

Rincon-Delgadillo, Paulina, OV

Robinson, Alex P. G., ON

Rowell, Kevin, 11

Rutigliani, Vito, OA

Sakita, Kyohei, $0 \mathrm{G}$

Santillan, Julius Joseph, OE, OF

Saouaf, $\mathrm{O} ., \mathrm{OQ}$

Sato, Hironobu, OU

Sato, Takashi, $1 \mathrm{~K}$

Schoofs, Stijn, 05

Seino, Yuriko, OU

Seshadri, Indira, 0

Seshimo, Takehiro, IF

Shamma, Nader, OA

Shen, Han-Ping, 1D

Shi, Heguang, $1 \mathrm{H}$

Shima, Motoyuki, 06

Shimada, Ryo, 06

Shimizu, Yoko, $1 \mathrm{~K}$

Shiozawa, Takahiro, 06

Shiraishi, Gosuke, 06

Shiraishi, Masayuki, OU

Shirakawa, Michihiro, OG

Singh, Lovejeet, 0 l

Sitterly, Jacob, 08, 1P

Sobieski, Daniel, OA

Soedibyo, Rio A., 12

Spence, Chris, 05

Stock, Hans-Jürgen, 06

Stokes, Harold, OJ, OM

Sullivan, Chris, OL

Tagawa, Seiichi, 06, $1 \mathrm{G}$

Takeshita, Kazuhiro, 06

Takigawa, Tomoaki, $1 \mathrm{~K}$

Tanaka, Yuji, OJ, OM

Tapily, Kanda, 11

Teyssèdre, H., 15

Timoshkov, Vadim, 05

Tiron, R., OQ

Tomono, Masaru, 06

Toriumi, Minoru, $0 \mathrm{E}, 13$

Tsuchihashi, Toru, OG

Tsukiyama, Koichi, OE, 13

Tsuzuki, Shuichi, 16

Umeda, Toru, 16

Vandenberghe, Geert, 06, 0C

Vandereyken, Jelle, 0J

van der Straten, Koen, OA

Vanelderen, Pieter, $0 \mathrm{C}$

Vesters, Yannick, OC

Viantka, Katja, OA

Wajda, Cory, 11

Wang, Chien-Wei, 10

Wang, Yu-Fu, 10

Weineck, Gerald, 1A
Welling, Ulrich, $1 \mathrm{~N}$

Weng, Ming-Hui, ID

Wise, Rich, OA

Wong, Sabrina, OL

Wu, Aiwen, 17, 18

Wu, Cheng-Han, 1D

Wu, Chieh-Han, 1D

Wu, Ping-Jui, 10

$X \cup$, Cheng Bai, 11

$X u, K u i, O W$

$\mathrm{Xu}$, Yongan, $\mathrm{Ol}$

Yaegashi, Hidetami, 05, IF

Yamada, Kazuki, IF

Yamada, Shintaro, OL

Yamamoto, Hiroki, IG

Yamamoto, Kimiko, OM

Yamashita, Fumiko, 05

Yamato, Masatoshi, IF

Yang, Dongxu, 1H

Yeung, Marco, 12

Yoshida, Keisuke, 06

Yoshihara, Kosuke, 06

Yoshimura, Shota, 05

Yoshino, Takumi, $\mathrm{OH}$

Yu, Fangzhou, OK

Zhou, Chun, OV

Zhou, Jianuo, 1E 
Proc. of SPIE Vol. 10586 1058601-10

Downloaded From: https://www.spiedigitallibrary.org/conference-proceedings-of-spie on 26 Apr 2023 Terms of Use: https://www.spiedigitallibrary.org/terms-of-use 


\section{Conference Committee}

Symposium Chair

Bruce W. Smith, Rochester Institute of Technology (United States)

Symposium Co-chair

Will Conley, Cymer LLC, an ASML company (United States)

Conference Chair

Christoph K. Hohle, Fraunhofer Institute for Photonic Microsystems (Germany)

Conference Co-chair

Roel Gronheid, KLA-Tencor/ ICOS Belgium (Belgium)

Conference Program Committee

Robert Allen, IBM Almaden Research Center (United States)

Gilles R. Amblard, SAMSUNG Austin Semiconductor LLC (United States)

Ramakrishnan Ayothi, JSR Micro, Inc. (United States)

Sean D. Burns, IBM Corporation (United States)

Ryan Callahan, FUJIFILM Electronic Materials U.S.A., Inc. (United States)

Ralph R. Dammel, EMD Performance Materials Corporation (United States)

Danilo De Simone, IMEC (Belgium)

Chao Fang, KLA-Tencor Texas (United States)

Douglas Guerrero, Brewer Science, Inc. (United States)

Clifford L. Henderson, University of South Florida (United States)

Craig D. Higgins, GLOBALFOUNDRIES Inc. (United States)

Scott W. Jessen, Texas Instruments Inc. (United States)

Yoshio Kawai, Shin-Ełsu Chemical Company, Ltd. (Japan)

Qinghuang Lin, IBM Thomas J. Watson Research Center (United States)

Nobuyuki N. Matsuzawa, Panasonic Corporation (Japan)

Steve S. Putna, Intel Corporation (United States)

Daniel P. Sanders, IBM Almaden Research Center (United States)

Mark H. Somervell, Tokyo Electron America, Inc. (United States)

Jason K. Stowers, Inpria (United States)

James W. Thackeray, Dow Electronic Materials (United States) 
Raluca Tiron, CEA-LETI (France)

Rick Uchida, Tokyo Ohka Kogyo America, Inc. (United States)

Thomas I. Wallow, ASML Brion Technologies (United States)

\section{Session Chairs}

1 Keynote Session

Christoph K. Hohle, Fraunhofer-Institut für Photonische Mikrosysteme (Germany)

Roel Gronheid, KLA-Tencor/ ICOS Belgium (Belgium)

2 EUV: Resist Processes: Joint session with conferences 10583 and 10586

Thomas I. Wallow, ASML Brion Technologies(United States)

James W. Thackeray, Dow Electronic Materials (United States)

3 EUV: Metal-based Resists: Joint session with conferences 10583 and 10586

Jason K. Stowers, Inpria Corporation (United States)

Christopher S. Ngai, Applied Materials, Inc. (United States)

4 Materials and Etch Integration: Joint session with conferences 10586

and 10589

Qinghuang Lin, IBM Thomas J. Watson Research Center (United States)

Sebastian U. Engelmann, IBM Thomas J. Watson Research Center (United States)

5 EUV: Fundamentals

Clifford L. Henderson, University of South Florida (United States)

Craig D. Higgins, GLOBALFOUNDRIES Inc. (United States)

$6 \quad$ EUV: Novel Processes

Danilo De Simone, IMEC (Belgium)

Rick Uchida, Tokyo Ohka Kogyo America, Inc. (United States)

7 Hardmasks and Underlayer

Douglas J. Guerrero, Brewer Science, Inc. (Belgium)

Ryan Callahan, FUJIFILM Electronic Materials U.S.A., Inc. (United States)

8 DSA Materials and Characterization: Joint session with conferences 10586 and 10584

Ralph R. Dammel, EMD Performance Materials Corporation (United States)

Chi-Chun Liu, IBM Corporation (United States) 
9 DSA Materials and Integration: Joint session with conferences 10586 and 10584

Daniel Sanders, IBM Research - Almaden (United States)

J. Alexander Liddle, National Institute of Standards and Technology (United States)

10 DSA: Defectivity and High-chi

Mark H. Somervell, Tokyo Electron America, Inc. (United States)

Ralph R. Dammel, EMD Performance Materials Corporation (United States)

11 DSA: Cylinder Forming BCP and Metrology

Ramakrishnan Ayothi, JSR Micro, Inc. (United States)

Raluca Tiron, CEA-LETI (France)

12 Novel Processes

Gilles R. Amblard, SAMSUNG Austin Semiconductor LLC (United States)

Nobuyuki N. Matsuzawa, Panasonic Corporation (Japan) 
Proc. of SPIE Vol. 10586 1058601-14

Downloaded From: https://www.spiedigitallibrary.org/conference-proceedings-of-spie on 26 Apr 2023 Terms of Use: https://www.spiedigitallibrary.org/terms-of-use 